\title{
Associação entre baixo peso ao nascer e asma: uma revisão sistemática da literatura
}

\author{
Moema N. Chatkin e Ana Maria B. Menezes²
}

Como citar Chatkin MN, Menezes AMB. Associação entre baixo peso ao nascer e asma: uma revisão sistemática da literatura. Rev Panam Salud Publica. 2005;17(2):102-9.

RESUMO Objetivo. Investigar a existência de associação entre baixo peso ao nascer $(<2500 \mathrm{~g})$ e asma. Resultados. As palavras-chave asthma, children, birth weight $e$ risk factors foram utilizadas para identificar estudos epidemiológicos analiticos em seres humanos, publicados em inglês, espanhol ou português entre 1990 e 2001, conforme as bases de dados MEDLINE (PubMed) e Literatura Latino-Americana e do Caribe em Ciências da Saúde (LILACS).

Foram identificados 41 artigos que tratavam da associação entre peso ao nascer e asma, sendo que, quanto ao delineamento, 21 eram longitudinais, 16 eram transversais e quatro eram estudos de caso-controle. O baixo peso ao nascer foi identificado como fator de risco para asma em 26 estudos. Dois estudos encontraram associação inversa entre o peso ao nascer e o risco de asma, e 15 não observaram uma associação entre esses fatores.

Conclusões. Permanece controversa a associação entre peso ao nascer e asma, devido, principalmente, à rede de fatores que concorrem para a determinação da asma. A associação entre baixo peso ao nascer e asma ocorre principalmente até os 5 anos, e diminui com o aumento da idade. A função pulmonar é um importante aspecto a ser considerado: as crianças nascidas com baixo peso podem ter a função pulmonar diminuída e, conseqüentemente, desenvolver asma; por outro lado, essas crianças podem ser asmáticas como decorrência do baixo peso, sem influência da função pulmonar. Nos seis estudos que avaliaram tanto as crianças de baixo peso ao nascer quanto as crianças de muito baixo peso ao nascer $(<1500 \mathrm{~g})$, este último fator implicou em risco maior para a asma. Finalmente, a contribuição de fatores intra-uterinos (como a nutrição) para o desenvolvimento de asma está ganhando importância na literatura. Em estudos futuros, o controle de possíveis fatores de confusão é essencial para o esclarecimento da relação entre peso ao nascer e asma.

Palavras chave Fatores de risco, grupos etários, peso ao nascer, prematuro, volume expiratório forçado.

A asma é uma doença comum na infância, e sua prevalência vem aumentando nas últimas décadas em diversos países $(1,2)$. Muitos estudos têm sido

1 Universidade Federal de Pelotas (UFPel), Departamento de Clínica Médica, e Secretaria Municipal de Saúde e Bem-Estar de Pelotas (RS), Brasil. Enviar correspondência para esta autora no seguinte endereço: Rua Gonçalves Chaves 3625/304, CEP 96015-560, Pelotas, RS, Brasil. E-mail: moema. chatkin@pelotas.com.br

2 UFPel, Departamento de Clínica Médica. realizados para identificar fatores de risco para a asma infantil; com freqüência, o baixo peso ao nascer $(<2500 \mathrm{~g})$ é apontado como um desses fatores de risco, tanto em associação com a prematuridade quanto como fator independente da idade gestacional. Além disso, o aumento do índice de sobrevivência de recém-nascidos com muito baixo peso ao nascer $(<1500 \mathrm{~g})$ tem sido associado à prevalência de asma e sintomas respiratórios na infância (3-5).

Outro ponto importante sugerido pela literatura é que os mecanismos de associação entre baixo peso ao nascer e asma podem ser diferentes para idades distintas (6). Svanes et al. (7) encontraram associação entre peso ao nascer e asma em uma coorte de adultos com idade entre 20 e 24 anos, mesmo após controle dos fatores de confusão. Essa 
associação entre baixo peso ao nascer e asma na vida adulta poderia indicar que o risco para asma é parcialmente estabelecido no início da vida. De acordo com a hipótese da origem fetal, o metabolismo e a estrutura orgânica do feto sofrem alterações em resposta à desnutrição intra-uterina, e essas alterações poderiam levar ao desenvolvimento de obstrução crônica ao fluxo aéreo na vida adulta (7-9).

Diversos mecanismos têm sido sugeridos para explicar a ocorrência de asma nas crianças com baixo peso ao nascer, entre eles:

- a redução da função pulmonar durante a infância (10);

- o tamanho diminuído dos pulmões ou a maior incidência de infecções virais durante a infância nas crianças de baixo peso ao nascer $(6,11)$;

- a imaturidade imunológica nos primeiros meses de vida e a exposição a alérgenos (12);

- e a hiper-reatividade brônquica $(13,14)$.

Embora ainda seja controversa, a associação entre asma e baixo peso ao nascer tem sido foco de poucos estudos. A maioria dos trabalhos estuda diversos fatores de risco para asma, podendo ou não incluir o peso ao nascer. Algumas pesquisas analisam apenas os recém-nascidos com muito baixo peso, sem compará-los com crianças com peso entre 1500 e $2499 \mathrm{~g}$ ao nascer.

Como o baixo peso ao nascer pode ser revertido com cuidados pré-natais adequados, o esclarecimento quanto à existência de uma associação entre baixo peso ao nascer e asma é muito importante. O objetivo do presente estudo foi revisar a literatura em busca de evidências contrárias ou favoráveis a essa associação.

\section{FONTE DOS DADOS}

Realizou-se uma revisão sistemática dos artigos publicados nas bases de dados MEDLINE (PubMed) e Literatura Latino-Americana e do Caribe em Ciências da Saúde (LILACS), utilizando-se as palavras-chave asthma, birth weight, children e risk factors.
Foram lidos os resumos de todos os artigos contendo as palavras-chave, sendo considerados como relevantes aqueles que mencionaram o estudo da associação entre peso ao nascer e asma ou sintomas de asma. Como a definição de asma varia entre os estudos, foram incluídos todos os que citaram a avaliação da associação entre peso ao nascer e asma ou sintomas de asma. Os demais critérios de inclusão foram: ser estudo epidemiológico analítico em seres humanos, ter sido publicado em espanhol, inglês ou português e ter sido publicado de janeiro de 1990 a outubro de 2002.

Ao realizar-se a busca, ficou evidente que os artigos abordavam três faixas etárias. Assim, embora não correspondam à divisão adotada pela Organização Mundial da Saúde (OMS), as seguintes faixas etárias foram consideradas: infância (crianças de até 5 anos); pré-adolescência (de 6 a 12 anos); e adolescência e fase adulta (mais de 12 anos).

\section{RESULTADOS}

Foram localizados 138 artigos, dos quais 44 foram selecionados como sendo relevantes a partir da leitura do resumo. Após a leitura dos 44 artigos na íntegra, 37 foram selecionados. Além disso, a leitura desses artigos levou à busca de mais 12 citações, das quais quatro foram selecionadas. Assim, foram revisados 41 artigos que tratavam da associação entre peso ao nascer e asma ou sintomas de asma.

Quanto ao delineamento, 16 estudos tinham delineamento transversal (11-13, 15-27); 21 eram estudos longitudinais $(1,4-7,28-43)$; e quatro eram estudos de caso-controle (44-47). Baixo peso ao nascer foi identificado como fator de risco para asma em 26 estudos, sendo que dois estudos encontraram associação inversa entre o peso ao nascer e o risco de asma, e 15 não encontraram essa associação (tabelas 1 e 2).

Os estudos utilizavam diversos critérios para definir o desfecho asma, entre os quais o diagnóstico de asma pelo médico, o relato de asma pelo paciente ou pelos pais, o chiado no peito e outros sintomas de asma, o uso de medicação broncodilatadora e a avaliação médica.

Trinta e três estudos utilizaram uma (ou ambas) das seguintes categorias de baixo peso ao nascer: muito baixo peso ao nascer, para bebês que nasceram com menos de 1500 g; e baixo peso ao nascer, para os que nasceram com peso entre 1500 e $2499 \mathrm{~g}$. Apenas oito estudos utilizaram categorias diferentes de peso ao nascer (em três o peso foi considerado como variável contínua, e em cinco foram considerados quartis, ou várias categorias).

Os diversos fatores implicados na associação entre baixo peso ao nascer e asma se inter-relacionam na determinação desta doença, ora como fatores de confusão, ora como mediadores da cadeia causal. Como resultado da revisão bibliográfica, no presente artigo será discutida a interação de três fatores: prematuridade, idade e função pulmonar.

\section{Prematuridade}

O peso ao nascer e a prematuridade parecem influenciar de formas diferentes a função pulmonar e o desenvolvimento da asma ou de seus sintomas, mesmo sendo o peso ao nascer fortemente dependente da idade gestacional. Rona et al. (15) encontraram associação entre idade gestacional e sibilância independentemente do peso ao nascer, assim como associação entre baixo peso ao nascer e déficit na função pulmonar independentemente da idade gestacional. Esses achados de associação de déficit de função pulmonar com baixo peso ao nascer após controle para idade gestacional sugerem que a nutrição intra-uterina pode ser um importante determinante de crescimento pós-natal da via aérea, sendo a função pulmonar afetada, principalmente, pelo ambiente intrauterino, enquanto que sintomas como sibilância na infância estariam mais relacionados com a prematuridade.

Alguns estudos têm demonstrado que a prematuridade propriamente dita, e não o retardo de crescimento intra-uterino, predispõe ao desenvolvimento de asma $(13,28)$. 
TABELA 1. Estudos publicados entre 1990 e 2001 que encontraram associação entre peso ao nascer e asma ou sintomas de asma ${ }^{a}$

\begin{tabular}{|c|c|c|c|c|c|}
\hline \multirow[b]{2}{*}{ Estudo } & \multirow[b]{2}{*}{ País } & \multirow[b]{2}{*}{ Delineamento } & \multicolumn{2}{|c|}{ Amostra } & \multirow[b]{2}{*}{ Resultados $^{b}$} \\
\hline & & & No. & Idade (anos) & \\
\hline Joseph et al. (21) & Estados Unidos & Transversal (escolar) & 126 & 6 a 8 & OR asma: $5,1(1,4 \text { a } 18,9)^{c}$ \\
\hline Palta et al. (32) & Estados Unidos & $\begin{array}{l}\text { Longitudinal (coorte } \\
\text { de nascimento) }\end{array}$ & 538 & 8 & $\begin{array}{l}\text { Prevalência da asma } \\
\text { - Muito baixo peso ao nascer: } 19 \% \\
\text { - Controles (> } 1500 \mathrm{~g}): 14 \%\end{array}$ \\
\hline Brooks et al. (3) & Estados Unidos & $\begin{array}{l}\text { Transversal } \\
\text { (populacional) }\end{array}$ & 807 & 13 & $\begin{array}{l}\text { Prevalência da asma } \\
\text { - Muito baixo peso ao nascer: } 21,9 \% \text {; OR: } 2,9 \\
\quad(2,3 \text { a } 3,6) \\
\text { - Baixo peso ao nascer: } 10,9 \% \text {; OR: } 1,4(1,1 \text { a } 1,8)\end{array}$ \\
\hline Rönmark et al. (1) & Suécia & Longitudinal (escolar) & 3339 & 7 a 8 & $\begin{array}{l}\text { OR prevalência asma }=2,9(1,8 \text { a } 4,8)^{c} \\
\text { OR incidência asma: } 6,7(2,9 \text { a } 15,2)^{\mathrm{c}} \\
\text { OR prevalência chiado: } 2,2(1,4 \text { a } 3,5)^{\mathrm{c}} \\
\text { OR incidência chiado: } 1,4(0,5 \text { a } 4,1)^{\mathrm{c}}\end{array}$ \\
\hline Gold et al. (4) & Estados Unidos & $\begin{array}{l}\text { Longitudinal (coorte } \\
\text { de nascimento) }\end{array}$ & 499 & 1 & $\mathrm{RR}=1,28(1,04 \text { a } 1,58)^{\mathrm{c}}$ \\
\hline $\begin{array}{l}\text { Leadbitter et al. } \\
\quad(37)\end{array}$ & Nova Zelândia & $\begin{array}{l}\text { Longitudinal (coorte } \\
\text { de nascimento) }\end{array}$ & 734 & 13 & $\begin{array}{l}\text { Prevalência da asma } \\
\text { - <2 } 500 \mathrm{~g}: 2,9 \% \\
\text { - } 2500 \text { a } 2900: 6,1 \% \\
\text { - } 3000 \text { a } 3400: 20,5 \% \\
\text { - } 3500 \text { a } 3900: 11,2 \% \\
\text { - } \geq 4000: 17,9 \% \\
\text { - < } 3000 \mathrm{~g} \\
\text { OR asma }=0,2(0,0 \text { a } 0,6)^{\mathrm{c}} \\
\text { OR asma atual }=0,2(0,0 \text { a } 0,6)^{c}\end{array}$ \\
\hline $\begin{array}{l}\text { Lewis e Britton } \\
\text { (35) }\end{array}$ & Reino Unido & $\begin{array}{l}\text { Longitudinal (coorte } \\
\text { de nascimento) }\end{array}$ & 6068 & 16 & $\begin{array}{l}\mathrm{OR}<2000 \mathrm{~g}: 1,84(0,99 \text { a } 3,40) \\
\mathrm{OR}<3000 \mathrm{~g}: 1,35(1,10 \text { a } 1,67)\end{array}$ \\
\hline Wjst et al. (11) & Alemanha & Transversal (escolar) & 2470 & 5 a 14 & $\begin{array}{l}\text { OR asma cumulativa: } 1,4(1,0 \text { a } 2,0)^{c} \\
\text { OR asma atual: } 1,6(1,0 \text { a } 2,6)^{\mathrm{c}}\end{array}$ \\
\hline Svanes et al. (7) & Noruega & $\begin{array}{l}\text { Longitudinal } \\
\text { (populacional) }\end{array}$ & 690 & 20 a 24 & OR chiado $1500 \mathrm{~g}$ ao nascer: $0,69(0,50$ a 0,95$)$ \\
\hline Slezak et al. (18) & Estados Unidos & $\begin{array}{l}\text { Transversal } \\
\text { (organização } \\
\text { governamental) }\end{array}$ & 1085 & 3 a 5 & $\begin{array}{l}\text { OR muito baixo peso ao nascer: } 4,7(0,9 \text { a } 26,1) \\
\text { OR baixo peso ao nascer: } 1,8(1,02 \text { a } 3,23)\end{array}$ \\
\hline Lewis et al. (24) & Reino Unido & $\begin{array}{l}\text { Transversal } \\
\text { (populacional) }\end{array}$ & $\begin{array}{c}11262 \\
(1974) \\
\text { e } 9266 \\
(1986)\end{array}$ & 16 & $\begin{array}{l}\text { Prevalência < } 2000 \mathrm{~g}=12,0 \% ; \mathrm{OR}=1,00 \\
\text { Prevalência } 2000 \text { a } 2500 \mathrm{~g}=6,8 \% ; \mathrm{OR}=0,77 \\
\text { Prevalência } 2500 \text { a } 3000 \mathrm{~g}=7,9 \% ; \mathrm{OR}=0,78 \\
\text { Prevalência }>3000 \mathrm{~g}=6,0 \% ; \mathrm{OR}=0,64 \\
P \text { tendência }=0,001\end{array}$ \\
\hline $\begin{array}{l}\text { Schaubel et al. } \\
\text { (30) }\end{array}$ & Canadá & $\begin{array}{l}\text { Longitudinal (coorte } \\
\text { retrospectiva) }\end{array}$ & 16207 & 4 & $\begin{array}{l}\text { OR muito baixo peso ao nascer }=2,1(1,1 \text { a } 4,0) \\
\text { OR baixo peso ao nascer }=1,2(0,9 \text { a } 1,7)\end{array}$ \\
\hline McLeod et al. (28) & Escócia & $\begin{array}{l}\text { Longitudinal (coorte } \\
\text { retrospectiva) }\end{array}$ & 866 & 8 a 9 & $\begin{array}{l}\text { OR uso de inalador: } 1,9(1,2 \text { a } 2,9)^{c} \\
\text { OR chiado: } 2,1(1,2 \text { a } 3,9)^{c}\end{array}$ \\
\hline
\end{tabular}


TABELA 1. (Continuação)

\begin{tabular}{|c|c|c|c|c|c|}
\hline \multirow[b]{2}{*}{ Estudo } & \multirow[b]{2}{*}{ País } & \multirow[b]{2}{*}{ Delineamento } & \multicolumn{2}{|c|}{ Amostra } & \multirow[b]{2}{*}{ Resultados $^{b}$} \\
\hline & & & No. & Idade (anos) & \\
\hline Azizi et al. (44) & Malásia & $\begin{array}{l}\text { Caso-controle } \\
\text { (hospitalar) }\end{array}$ & 359 & $\begin{array}{c}1 \text { mês a } 5 \\
\text { anos }\end{array}$ & OR: $2,2(1,1 \text { a } 4,4)^{c}$ \\
\hline Lewis et al. (6) & Inglaterra & $\begin{array}{l}\text { Longitudinal (coorte } \\
\text { de nascimento) }\end{array}$ & 15712 & 5 e 16 & OR: $1,26(1,07 \text { a } 1,50)^{c}$ \\
\hline Arshad et al. (29) & Reino Unido & $\begin{array}{l}\text { Longitudinal (coorte } \\
\text { de nascimento) }\end{array}$ & 1174 & 4 & OR: $3,0(1,4 \text { a } 6,5)^{c}$ \\
\hline $\begin{array}{l}\text { Von Mutius et al. } \\
\text { (17) }\end{array}$ & Alemanha & Transversal (escolar) & 5030 & 9 a 11 & OR meninas prematuras: $2,6(1,4$ a 4,7$)$ \\
\hline $\begin{array}{l}\text { Frischer et al. } \\
\text { (22) }\end{array}$ & Alemanha & Transversal (escolar) & 1812 & 8 & $\begin{array}{l}\text { OR asma: } 1,57 ; P<0,09^{c} \\
\text { OR tosse pós-exercício: } 2,09 ; P<0,02^{c}\end{array}$ \\
\hline $\begin{array}{l}\text { Seidman et al. } \\
\text { (12) }\end{array}$ & Israel & $\begin{array}{l}\text { Transversal } \\
\text { (recrutas) }\end{array}$ & 20312 & 17 & $\begin{array}{l}\text { OR } 2000 \text { a } 2499 \text { g: } 1,49(1,05 \text { a } 2,12) \\
\text { OR < } 2000 \text { g: } 1,44(0,79 \text { a } 2,61)\end{array}$ \\
\hline $\begin{array}{l}\text { Greenough et al. } \\
\quad(45)\end{array}$ & Reino Unido & $\begin{array}{l}\text { Caso-controle } \\
\text { (hospitalar) }\end{array}$ & 151 & 1 & $\begin{array}{l}\text { Prevalência de sintomas para baixo peso ao nascer } \\
\text { contra peso não baixo ao nascer: } 51 \text { vs. } 31 \% \text {; } \\
P<0,01\end{array}$ \\
\hline $\begin{array}{l}\text { Schwartz et al. } \\
\text { (20) }\end{array}$ & Estados Unidos & $\begin{array}{l}\text { Transversal } \\
\text { (populacional) }\end{array}$ & 5672 & $\begin{array}{l}6 \text { meses } \\
\text { a } 11 \text { anos }\end{array}$ & $\begin{array}{l}\text { OR asma }=1,31(1,00 \text { a } 1,72)^{c} \\
\text { OR chiado }=1,33\left(1,08 \text { a } 1,65^{c}\right.\end{array}$ \\
\hline $\begin{array}{l}\text { Weitzman et al. } \\
\text { (19) }\end{array}$ & Estados Unidos & $\begin{array}{l}\text { Transversal } \\
\text { (populacional) }\end{array}$ & 15416 & 0 a 17 & OR asma $=2,0(1,1 \text { a } 3,5)^{c}$ \\
\hline
\end{tabular}

a Conforme Medline (PubMed) e LILACS.

${ }^{\mathrm{b}}$ Em todos os estudos, baixo peso ao nascer < $2500 \mathrm{~g}$; e muito baixo peso ao nascer < $1500 \mathrm{~g}$.

${ }^{\mathrm{c}}$ Medidas de efeito da ocorrência do desfecho em crianças de baixo peso em comparação a crianças com peso normal.

Von Mutius et al. (17) estudaram o baixo peso ao nascer somente em combinação com a prematuridade e encontraram associação com asma atual em meninas de 9 a 11 anos. Outro estudo, realizado em uma coorte representativa da população da Inglaterra, encontrou associação do baixo peso ao nascer, mas não da idade gestacional, com sintomas de asma em crianças aos 5 anos, sendo que $85 \%$ dessas crianças deixaram de ter asma aos 16 anos (6).

Seis estudos $(3,5,18,24,30,35)$ consideraram, ainda, as duas categorias de peso ao nascer: baixo peso $(<2500 \mathrm{~g})$ e muito baixo peso $(<2000$ ou $1500 \mathrm{~g}$ ). A figura 1 mostra que, em quase todos esses estudos, o risco de asma foi cerca de 2 vezes maior para as crianças com peso $<1500 \mathrm{~g}$.

\section{Idade}

Foram identificadas três faixas etárias de interesse na associação entre peso ao nascer e asma: infância (até 5 anos), pré- adolescência (mais de 5 a 12 anos) e fase adulta (mais de 12 anos). Todos os nove estudos que analisaram a faixa etária infantil $(3,4,18-20,29,30,44,45)$ encontraram associação entre baixo peso ao nascer e asma, com razão de chances (odds ratio, OR) variando de 1,2 a 4,7. Quando as crianças de muito baixo peso ao nascer foram estudadas isoladamente, o risco de asma ou de sintomas de asma foi 2 vezes maior do que nas crianças de baixo peso ao nascer. Isso poderia ser uma conseqüência da obstrução persistente das pequenas vias aéreas nas crianças prematuras que desenvolveram displasia broncopulmonar.

Lewis et al. (6) constataram que o baixo peso ao nascer e o fumo na gestação foram fatores de risco para sibilância aos 5 anos; porém, em 85\% dessas crianças, os sintomas desapareceram até os 16 anos. Isso sugere que há dois tipos de processo envolvidos na ocorrência de sibilância na infância: um relacionado com as pequenas vias aéreas, precipitado por infecções virais e associado ao baixo peso ao nascer e ao fumo materno; e outro que persiste na adolescência e que poderia ser a expressão de uma doença alérgica.

Entre os estudos que avaliaram crianças na pré-adolescência, nove encontraram uma associação entre baixo peso ao nascer e asma $(1,6,11,17,21$, $22,28,32,33)$, enquanto que cinco não observaram essa associação $(13,15,23$, $31,46)$. Um estudo realizado na Alemanha, cujo objetivo foi examinar o efeito do baixo peso ao nascer e da prematuridade sobre os sintomas respiratórios e a função pulmonar, identificou o baixo peso ao nascer como sendo mais importante do que a prematuridade na determinação de doença respiratória dos 5 aos 14 anos (11).

Barker et al. (9) sugeriram que o efeito a longo prazo do baixo peso ao nascer na função pulmonar do adulto é uma forma de "programação", ou seja, as alterações ocorridas no feto em decorrência da desnutrição no ambiente intra-uterino seriam responsáveis pelo desenvolvimento de obstrução crônica ao fluxo aéreo. Svanes et al. (7), que 
TABELA 2. Estudos publicados entre 1990 e 2001 que não encontraram associação entre peso ao nascer e asma ou sintomas de asma ${ }^{a}$

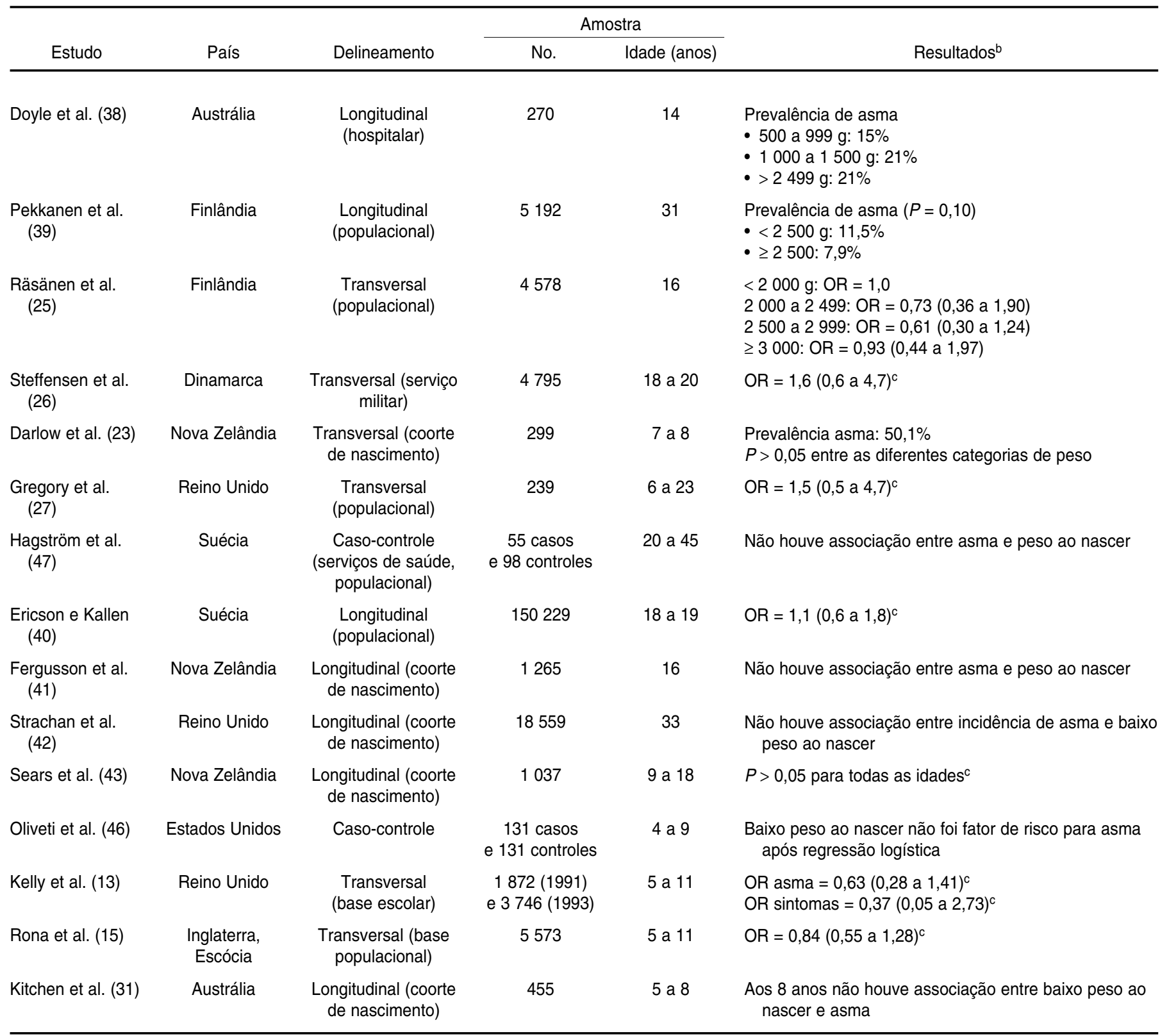

a Conforme Medline (PubMed) e LILACS.

${ }^{\mathrm{b}}$ Em todos os estudos, baixo peso ao nascer $<2500 \mathrm{~g}$; e muito baixo peso ao nascer $<1500 \mathrm{~g}$.

${ }^{c}$ Medidas de efeito da ocorrência do desfecho em crianças de baixo peso em comparação a crianças com peso normal.

estudaram uma coorte dos 20 aos 24 anos, sugeriram que a associação de baixo peso ao nascer e asma não resulta de alergia, e também que o risco de asma é estabelecido no início da vida.

Apenas oito dos 18 estudos na faixa etária adulta encontraram associação entre baixo peso ao nascer e asma $(5,7$, 12, 24, 34-37), sendo que, em dois deles $(36,37)$, o baixo peso ao nascer foi fator de proteção para asma ou sintomas de asma. Em 10 estudos não foi encontrada uma associação entre baixo peso ao nascer e asma (25-27, 38-43, 47).

Seidman et al. (12), que estudaram a associação de baixo peso ao nascer e incidência de asma em uma grande amostra durante 17 anos, demonstraram que as crianças que nasceram com menos de $2500 \mathrm{~g}$ estiveram sujeitas a um risco $40 \%$ maior de ter asma diagnosticada aos 17 anos de idade.

Shaheen et al. (5), estudando uma coorte de nascimento na Grã-Bretanha aos 26 anos, observaram um risco de asma quase 2 vezes maior entre os que haviam nascido com menos de $2000 \mathrm{~g}$, e um risco 1,5 vez maior para os indivíduos com peso ao nascer 
FIGURA 1. Risco de asma entre crianças nascidas com baixo peso ou muito baixo peso conforme estudos publicados entre 1990 e $2001^{\text {a }}$

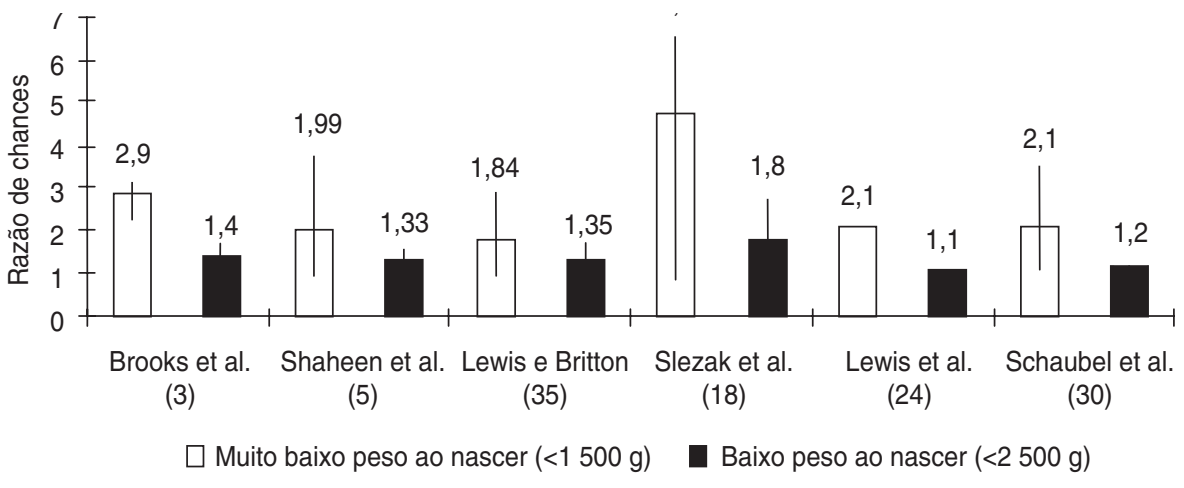

a Medline (PubMed) e LILACS.

entre 2000 e $2500 \mathrm{~g}$, tendo os resultados sido ajustados para fatores de confusão e para o índice de massa corporal (IMC) atual.

\section{Função pulmonar}

A associação do baixo peso ao nascer com a diminuição da função pulmonar (volume expiratório forçado no $1^{\circ}$ segundo) e a morte por doença pulmonar obstrutiva crônica em adultos (9) sugere que fatores intra-uterinos, tais como a nutrição, são importantes determinantes do crescimento pósnatal da via aérea.

A introdução da ventilação assistida em 1960 e da terapia surfactante em 1990, e o uso de esteróides pré-natais, além das novas estratégias de ventilação, resultaram em maior sobrevivência dos recém-nascidos com menos de $1500 \mathrm{~g} \mathrm{e}$, conseqüentemente, no aumento da prevalência de displasia broncopulmonar (48), com déficit na função pulmonar transitória ou permanente, o que poderia levar a sintomas de asma na infância ou na vida adulta.

Existem evidências da associação do baixo peso ao nascer com a diminuição da função pulmonar já na infância (15, 31), embora não haja consenso (49). Alguns estudos demonstraram a existência de associação entre a função pulmonar diminuída na infância e a prevalência de sintomas respiratórios (31). Kennedy et al. (50), em estudo que buscou avaliar a importância da contribuição do baixo peso ao nascer, da idade gestacional, da doença respiratória neonatal e de seu tratamento para a função pulmonar subseqüente em uma coorte de crianças nascidas com menos de $1500 \mathrm{~g}$, verificaram que o peso ao nascer teve maior contribuição para a função pulmonar do que a idade gestacional, mas a suplementação de oxigênio foi o fator preditivo de maior significância. Já Chan et al. (10) demonstraram que, aos 9 anos, a associação entre déficit na função respiratória em crianças de baixo peso ao nascer é independente da doença respiratória neonatal e do uso de oxigênio ou de ventilação mecânica, sendo a prematuridade e outros fatores constitucionais melhores preditores da função pulmonar (10).

Wjst et al. (11), estudando escolares de 5 a 14 anos na Alemanha, encontraram redução no tamanho pulmonar e no fluxo aéreo, assim como hiperreatividade brônquica aumentada, nas crianças de baixo peso não prematuras. O efeito do baixo peso ao nascer diminuiu com o aumento da idade, e houve maior prevalência de diagnóstico de asma entre as crianças de baixo peso ao nascer não prematuras.

\section{CONCLUSÕES}

Apesar de serem diversos os fatores implicados na associação estudada, eles se inter-relacionam na determi- nação da asma, ora como fatores de confusão, ora como mediadores da cadeia causal. Por exemplo, estudos têm demonstrado que o baixo nível socioeconômico pode estar associado com a ocorrência de asma como fator independente ou como fator que origina maus cuidados pré-natais $(19,51-53)$. Além disso, o baixo nível socioeconômico está associado ao fumo materno e pode levar, juntamente com este fator, à prematuridade $(19,54)$. A menor idade da mãe, juntamente com fumo, maus cuidados pré-natais e baixa idade gestacional, poderia resultar em baixo peso ao nascer e função pulmonar diminuída e, conseqüentemente, em asma $(19,55)$. O sexo masculino e a raça negra são independentemente associados à asma e ao baixo peso ao nascer em diversos estudos, e também podem estar implicados nessa rede, assim como a doença respiratória neonatal, que é fortemente associada ao baixo peso ao nascer, à prematuridade e à asma $(6,20)$. Desse modo, o peso ao nascer pode ser um fator mediador na cadeia causal ou um fator de risco independente para asma.

Analisando-se a literatura, é possível concluir que a associação entre baixo peso ao nascer e asma é mais comum na infância. É interessante ressaltar que esse efeito diminui progressivamente à medida que aumenta a idade: na infância, todos os estudos encontrados na revisão apontaram o baixo peso ao nascer como fator de risco para asma; já na pré-adolescência, $64,3 \%$ dos estudos encontraram associação entre baixo peso ao nascer e asma, enquanto os restantes $35,7 \%$ não observaram essa associação. Na idade adulta, esses percentuais se inverteram, ou seja, a maioria dos estudos não encontrou associação entre baixo peso ao nascer e asma. De fato, um terço deles encontrou associação e $11,1 \%$ demonstraram um efeito contrário do baixo peso ao nascer em relação à asma-um efeito protetor. Evidentemente, os fatores de confusão na cadeia são bem maiores em se tratando de adultos, o que dificulta a interpretação dos achados. Cabe ressaltar que o número de estudos encontrados ainda é pequeno, o que impede a generalização dos resultados. Outra limi- 
tação a ser levada em conta é que alguns dos estudos não controlaram certos fatores de confusão, como idade gestacional e fumo, o que pode ter importantes implicações para os resultados. Tentou-se averiguar se os achados poderiam ser mais conclusivos avaliando apenas aqueles estudos em que pelo menos a idade gestacional foi controlada, mas não houve diferenças nas conclusões aqui mencionadas. A análise estatística controlando os possíveis fatores de confusão de níveis superiores ao baixo peso ao nascer é essencial para o esclarecimento dessa complexa rede multifatorial de causação da asma.

Outro aspecto importante é o papel da função pulmonar na associação entre asma e baixo peso ao nascer, ou seja: as crianças nascidas com baixo peso podem ter função pulmonar diminuída e, conseqüentemente, desenvolver quadros de asma; por outro lado, essas crianças podem ser asmáticas apenas pelo baixo peso ao nascer, sem que a função pulmonar tenha um papel mediador nessa associação. Não se pode afirmar, a partir dos estudos já publicados, até que ponto a função pulmonar está diretamente associada ao baixo peso ao nascer e, conseqüentemente, à asma.

Quanto à relação entre o muito baixo peso ao nascer e a asma, a literatura é mais consistente. A maioria dos estudos de comparação de crianças de baixo peso ao nascer com crianças de muito baixo peso ao nascer constatou que este último fator implicava em risco maior para a asma do que o primeiro. Novamente, fica a indagação sobre até que ponto a função pulmonar poderia estar interferindo ou mediando essa associação.

Finalmente, a contribuição de fatores intra-uterinos (tais como a nutrição) para o desenvolvimento de asma parece estar ganhando cada vez mais importância na literatura. Estudos longitudinais que abordem esse tema, com um melhor controle dos possíveis fatores de confusão, são essenciais para esclarecer o papel do baixo peso ao nascer no desencadeamento da asma.

\section{REFERÊNCIAS}

1. Ronmark E, Jonsson E, Platts-Mills T, Lundback $B$. Incidence and remission of asthma in schoolchildren: report from the obstructive lung disease in northern Sweden studies. Pediatrics. 2001;107(3):E37.

2. Kuehni CE, Davis A, Brooke AM, Silverman $\mathrm{M}$. Are all wheezing disorders in very young (preschool) children increasing in prevalence? Lancet. 2001;357(9271):1821-5.

3. Brooks AM, Byrd RS, Weitzman M, Auinger $P$, McBride JT. Impact of low birth weight on early childhood asthma in the United States. Arch Pediatr Adolesc Med. 2001;155(3): 401-6.

4. Gold DR, Burge HA, Carey V, Milton DK, Platts-Mills T, Weiss ST. Predictors of repeated wheeze in the first year of life: the relative roles of cockroach, birth weight, acute lower respiratory illness, and maternal smoking. Am J Respir Crit Care Med. 1999;160(1): 227-36.

5. Shaheen SO, Sterne JA, Montgomery SM, Azima $\mathrm{H}$. Birth weight, body mass index and asthma in young adults. Thorax. 1999;54(5): 396-402.

6. Lewis S, Richards D, Bynner J, Butler N, Britton J. Prospective study of risk factors for early and persistent wheezing in childhood. Eur Respir J. 1995;8(3):349-56.

7. Svanes C, Omenaas E, Heuch JM, Irgens LM, Gulsvik A. Birth characteristics and asthma symptoms in young adults: results from a population-based cohort study in Norway. Eur Respir J. 1998;12(6):1366-70.

8. Stein CE, Kumaran K, Fall CHD, Shaheen SO, Osmond C, Barker DJP. Relation of fetal growth to adult lung function in South India. Thorax. 1997;52(10):895-9.

9. Barker DJP, Godfrey KM, Fall C, Osmond C, Winter PD, Shaheen SO. Relation of birth weight and childhood respiratory infection to adult lung function and death from chronic obstructive airways disease. Br Med J. 1991; 303(6804):671-5

10. Chan KN, Wong YC, Silverman M. Relationship between infant lung mechanics and childhood lung function in children of very low birth weight. Pediatr Pulmonol. 1990;8(2): 74-81.

11. Wjst M, Popescu M, Trepka MJ, Heinrich J, Wichmann HE. Pulmonary function in children with initial low birth weight. Pediatr Allergy Immunol. 1998;9(2):80-90.

12. Seidman DS, Laor A, Gale R, Stevenson DK, Danon YL. Is low birth weight a risk factor for asthma during adolescence? Arch Dis Child. 1991;66(5):584-7.

13. Kelly YJ, Brabin BJ, Milligan P, Heaf DP, Reid J, Pearson MG. Maternal asthma, premature birth, and the risk of respiratory morbidity in schoolchildren in Merseyside. Thorax. 1995; 50(5):525-30.

14. Bertrand JM, Riley SP, Popkins J, Coates AL. The long-term pulmonary sequelae of prematurity: the role of familial airway hyperreactivity and the respiratory distress syndrome. N Engl J Med. 1985;312(12):742-5.

15. Rona RJ, Gulliford MC, Chinn S. Effects of prematurity and intrauterine growth on respiratory health and lung function in childhood. Br Med J. 1993;306(6881):817-20.

16. Chan KN, Elliman A, Bryan E, Silverman M. Clinical significance of airway responsiveness in children of low birthweight. Pediatr Pulmonol. 1989;7(4):251-8.

17. von Mutius E, Nicolai T, Martinez FD. Prematurity as a risk factor for asthma in preadolescent children. J Pediatr. 1993;123(2):223-9.

18. Slezak JA, Persky VW, Kviz FJ, Ramakrishnan $\mathrm{V}$, Byers C. Asthma prevalence and risk factors in selected Head Start sites in Chicago. J Asthma. 1998;35(2):203-12.

19. Weitzman M, Gortmaker S, Sobol A. Racial, social, and environmental risks for childhood asthma. Am J Dis Child. 1990;144(11):1189-94.

20. Schwartz J, Gold D, Dockery DW, Weiss ST, Speizer FE. Predictors of asthma and persistent wheeze in a national sample of children in the United States. Association with social class, perinatal events, and race. Am Rev Respir Dis. 1990;142(3):555-62.

21. Joseph CL, Ownby DR, Peterson EL, Johnson CC. Does low birth weight help to explain the increased prevalence of asthma among African-Americans? Ann Allergy Asthma Immunol. 2002;88(5):507-12.

22. Frischer T, Kuehr J, Meinert R, Karmaus W, Barth R, Hermann-Kunz E, et al. Relationship between low birth weight and respiratory symptoms in a cohort of primary school children. Acta Paediatr. 1992;81(12):1040-1.

23. Darlow BA, Horwood LJ, Mogridge N. Very low birthweight and asthma by age seven years in a national cohort. Pediatr Pulmonol. 2000;30(4):291-6.

24. Lewis S, Butland B, Strachan D, Bynner J, Richards D, Butler N, et al. Study of the aetiology of wheezing illness at age 16 in two national British birth cohorts. Thorax. 1996; 51(7):670-6.

25. Räsänen $\mathrm{M}$, Kaprio J, Laitinen $\mathrm{T}$, Winter $\mathrm{T}$, Koskenvuo M, Laitinen LA. Perinatal risk factors for asthma in Finnish adolescent twins. Thorax. 2000;55(1):25-31.

26. Steffensen FH, Sorensen HT, Gillman MW, Rothman KJ, Sabroe S, Fischer P, et al. Low birth weight and preterm delivery as risk factors for asthma and atopic dermatitis in young adult males. Epidemiology. 2000;11(2):185-8.

27. Gregory A, Doull I, Pearce N, Cheng S, Leadbitter $\mathrm{P}$, Holgate $\mathrm{S}$, et al. The relationship between anthropometric measurements at birth: asthma and atopy in childhood. Clin Exp Allergy. 1999;29(3):330-3.

28. McLeod A, Ross P, Mitchell S, Tay D, Hunter L, Hall A, et al. Respiratory health in a total very low birthweight cohort and their classroom controls. Arch Dis Child. 1996;74(3): 188-94.

29. Arshad SH, Stevens M, Hide DW. The effect of genetic and environmental factors on the prevalence of allergic disorders at the age of two years. Clin Exp Allergy. 1993;23(6):504-11. 
30. Schaubel D, Johansen H, Dutta M, Desmeules M, Becker A, Mao Y. Neonatal characteristics as risk factors for preschool asthma. J Asthma. 1996:33(4):255-64.

31. Kitchen WH, Olinsky A, Doyle LW, Ford GW, Murton LJ, Slonim L, et al. Respiratory health and lung function in 8-year-old children of very low birth weight: a cohort study. Pediatrics. 1992;89(6 Pt 2):1151-8.

32. Palta M, Sadek-Badawi M, Sheehy M, Albanese A, Weinstein M, McGuinness G, et al. Respiratory symptoms at age 8 years in a cohort of very low birth weight children. Am J Epidemiol. 2001;154(6):521-9.

33. Annesi-Maesano I, Moreau D, Strachan D. In utero and perinatal complications preceding asthma. Allergy. 2001;56(6):491-7.

34. Braback L, Hedberg A. Perinatal risk factors for atopic disease in conscripts. Clin Exp Allergy. 1998;28(8):936-42.

35. Lewis SA, Britton JR. Consistent effects of high socioeconomic status and low birth order, and the modifying effect of maternal smoking on the risk of allergic disease during childhood. Respir Med. 1998;92(10):1237-44.

36. Withers NJ, Low L, Holgate ST, Clough JB. The natural history of respiratory symptoms in a cohort of adolescents. Am J Respir Crit Care Med. 1998;158(2):352-7.

37. Leadbitter P, Pearce N, Cheng S, Sears MR, Holdaway MD, Flannery EM, et al. Relationship between fetal growth and the development of asthma and atopy in childhood. Thorax. 1999;54(10):905-10.

38. Doyle LW, Cheung MM, Ford GW, Olinsky A, Davis NM, Callanan C. Birth weight $<1501 \mathrm{~g}$ and respiratory health at age 14 . Arch Dis Child. 2001;84(1):40-4.
39. Pekkanen J, Xu B, Jarvelin MR. Gestational age and occurrence of atopy at age 31-a prospective birth cohort study in Finland. Clin Exp Allergy. 2001;31(1):95-102.

40. Ericson A, Kallen B. Very low birthweight boys at the age of 19. Arch Dis Child Fetal Neonatal Ed. 1998;78(3):F171-4.

41. Fergusson DM, Crane J, Beasley R, Horwood LJ. Perinatal factors and atopic disease in childhood. Clin Exp Allergy. 1997;27(12): 1394-1401.

42. Strachan DP, Butland BK, Anderson HR. Incidence and prognosis of asthma and wheezing illness from early childhood to age 33 in a national British cohort. BMJ. 1996;312(7040): 1195-9.

43. Sears MR, Holdaway MD, Flannery EM, Herbison GP, Silva PA. Parental and neonatal risk factors for atopy, airway hyperresponsiveness, and asthma. Arch Dis Child. 1996;75(5):392-8.

44. Azizi BH, Zulkifli HI, Kasim S. Indoor air pollution and asthma in hospitalized children in a tropical environment. J Asthma. 1995;32(6): 413-8.

45. Greenough A, Maconochie I, Yuksel B. Recurrent respiratory symptoms in the first year of life following preterm delivery. J Perinat Med. 1990;18(6):489-94.

46. Oliveti JF, Kercsmar CM, Redline S. Pre- and perinatal risk factors for asthma in inner city African-American children. Am J Epidemiol. 1996;143(6):570-7.

47. Hagstrom B, Nyberg P, Nilsson PM. Asthma in adult life-is there an association with birth weight? Scand J Prim Health Care. 1998;16(2): $117-20$.
48. Kennedy JD. Lung function outcome in children of premature birth. J Paediatr Child Health. 1999;35(6):516-21.

49. Matthes JWA, Lewis PA, Davies DP, Bethel JA. Birth weight at term and lung function in adolescence: no evidence for a programmed effect. Arch Dis Child. 1995;73(3):231-4.

50. Kennedy JD, Edward LJ, Bates DJ, Martin AJ, Dip SN, Haslam RR, et al. Effects of birthweight and oxygen supplementation on lung function in late childhood in children of very low birth weight. Pediatr Pulmonol. 2000; 30(1): 32-40.

51. Weitzman M, Gortmaker SL, Sobol AM, Perrin JM. Recent trends in the prevalence and severity of childhood asthma. JAMA. 1992 268(19):2673-7.

52. Gergen PJ, Mullaly DI, Evans R. National survey of prevalence of asthma among children in the United States, 1976 to 1980. Pediatrics. 1988;81(1):1-7.

53. Halfon N, Newacheck PW. Childhood asthma and poverty: differential impacts and utilization of health services. Pediatrics. 1993;91(1): 56-61.

54. Oliveti JF, Kercsmar CM, Redline S. Pre and perinatal risk factors for asthma in inner city African-American children. Am J Epidemiol. 1996;143(6):570-7.

55. Infante-Rivard C. Young maternal age: a risk factor for childhood asthma? Epidemiology. 1995;6(2):178-80.

Manuscrito recebido em 3 de setembro de 2003. Aceito em versão revisada em 24 de agosto de 2004.
ABSTRACT

\section{The association between low birthweight and asthma: a systematic literature review}

Objective. To determine if there is an association between low birthweight $(<2500 \mathrm{~g})$ and asthma.

Results. The key words "asthma," "children," "birth weight," and "risk factors" were used to identify analytical epidemiological studies on humans that were published in English, Portuguese, or Spanish between 1990 and 2001 and that were indexed in either of two bibliographic databases: MEDLINE (PubMed) and Latin American and Caribbean Health Sciences (Literatura Latino-Americana e do Caribe em Ciências da Saúde (LILACS)).

We identified 41 articles dealing with the association between birthweight and asthma: 21 were longitudinal studies, 16 were cross-sectional studies, and 4 were case-control studies. Low birthweight was identified as a risk factor for asthma in 26 of the articles. Two studies found an inverse association between birthweight and risk for asthma, and 15 did not find any association.

Conclusions. The association between birthweight and asthma remains controversial, mainly due to the extensive network of factors that contribute to the development of asthma. The association between low birthweight and asthma occurs mainly among children up to 5 years of age, and it decreases as age increases. Pulmonary function is an important factor that needs to be considered. Low birthweight children may have decreased pulmonary function and consequently develop asthma. On the other hand, these children may have asthma as a consequence of low birthweight, without pulmonary function being a factor. In the six studies comparing low birthweight children $(<2500 \mathrm{~g})$ with very low birthweight children $(<1500 \mathrm{~g})$, very low birthweight was associated with a greater risk for developing asthma. Finally, the contribution of intrauterine factors (such as nutrition) in the development of asthma is receiving increasing attention in the scientific literature. Controlling for possible confounding factors is essential to clarifying the relationship between birthweight and asthma.

Keywords: Risk factors; age groups; birth weight; infant, premature; forced expiratory volume. 